\title{
VALIDAÇÃO DO MAPGEO2010 E COMPARAÇÃO COM MODELOS DO GEOPOTENCIAL RECENTES
}

Validation of the MAPGEO2010 and comparison with recent geopotential models

\author{
ANA CRISTINA OLIVEIRA CANCORO DE MATOS ${ }^{1}$ \\ DENIZAR BLITZKOW ${ }^{1}$ \\ GABRIEL DO NASCIMENTO GUIMARÃES ${ }^{1}$ \\ MARIA CRISTINA BARBOSA LOBIANCO ${ }^{2}$ \\ SÔNIA MARIA ALVES COSTA ${ }^{2}$ \\ ${ }^{1}$ Escola Politécnica da Universidade de São Paulo \\ Laboratório de Topografia e Geodésia \\ EPUSP-PTR \\ ${ }^{2}$ Instituto Brasileiro de Geografia e Estatística \\ CGED-Coordenação de Geodésia \\ IBGE/DGC - Diretoria de Geociências \\ acocmatos@gmail.com; dblitzkow@usp.br; \\ gabrielguimaraes33@hotmail.com;sonia.lves@ibge.gov.br; lobianco@ibge.gov.br
}

\section{RESUMO}

Este trabalho visa apresentar a metodologia de cálculo e analisar o resultado do modelo de alturas geoidais para o Brasil. $\mathrm{O}$ mesmo abrange a área compreendida pelas latitudes de $6^{\circ} \mathrm{N}$ e $35^{\circ} \mathrm{S}$ e pelas longitudes de $75^{\circ} \mathrm{W}$ e $30^{\circ} \mathrm{W}$. Os dados gravimétricos terrestres foram atualizados com as mais recentes medições no Brasil e seus países vizinhos. As anomalias completas de Bouguer e de Helmert sobre a superfície terrestre foram calculadas através do pacote canadense SHGEO. A componente de curto comprimento de onda foi estimada através da integral modificada de Stokes utilizando a técnica FFT. O modelo do geopotencial EGM2008 serviu como modelo de referência restrito ao grau e ordem 150. Foram utilizadas 844 observações GPS sobre a rede de nivelamento geométrico para comparar com o modelo geoidal obtido. As anomalias de altura adicionadas de um termo de correção dependente da topografia derivadas do EGM2008 (grau 2190 e ordem 2159), GO_CONS_GCF_2_DIR_R2 (grau e ordem 240), GOCO02S (grau e ordem 250), EIGEN 51C (grau e ordem 359) e EIGEN 6C (grau e ordem 1420), bem como as alturas geoidais derivadas do MAPGEO2004 (antigo modelo oficial do IBGE) também foram comparadas com os pontos GPS sobre nivelamento. 
Palavras-Chave: Geoide; Geodésia; GPS; Nivelamento Geométrico; Altitude; Brasil.

\begin{abstract}
This paper presents the offorts to calculate the geoid model for Brazil. It is limited by $6^{\circ} \mathrm{N}$ and $35^{\circ} \mathrm{S}$ in latitude and $30^{\circ} \mathrm{W}$ and $75^{\circ} \mathrm{W}$ in longitude. The terrestrial gravity data for the continent have been updated by means of the most recent surveys in Brazil and in the neighbour countries. The complete Bouguer and Helmert gravity anomalies have been derived through the Canadian package SHGEO. The short wavelength component was estimated via FFT. The geopotential model EGM2008 was used as a reference field restricted to degree and order 150. The model was validated over 844 GPS observations on Bench Marks of the spirit leveling network. The height anomalies plus a topography dependent correction term derived from EGM2008 (degree 2190 and order 2159), GO_CONS_GCF_2_DIR_R2 (degree and order 240), GOCO02S (degree and order 250), EIGEN 51C (degree and order 359) and EIGEN 6C (degree and order 1420), geoidal height derived from MAPGEO2004 (old official geoid model in Brazil) have also been compared to the GPS points on Bench Marks.
\end{abstract}

Keywords: Geoid; Geodesy; GPS; Spirit Levelling; Height; Brazil

\title{
1. INTRODUÇÃO
}

Desde a publicação do MAPGEO2004, o Laboratório de Topografia e Geodésia da Escola Politécnica da USP - LTG/EPUSP trabalha em parceiras com outras instituições visando a densificação gravimétrica em território nacional e a implantação de redes gravimétricas em algumas regiões da América do Sul com objetivo de calcular um modelo geoidal mais consistente que o anterior. Com isso, no ano de 2010 foi calculado o mais recente modelo de alturas geoidais para o país, o MAPGEO2010.

Dentro dos esforços recentes, os dados terrestres de gravimetria para o continente foram atualizados com os levantamentos ocorridos nos últimos anos na Argentina, no Brasil, no Equador e no Paraguai. Todas essas novas informações foram validadas utilizando o programa denominado DIVA, desenvolvido por Michel Sarrailh no Bureau Gravimétrique International (BGI) que tem sede na cidade de Toulouse, França. O Modelo Digital de Terreno (MDT) utilizado foi o SAM3s_v2 (BLITZKOW et al., 2008a,b), o qual baseia-se no SRTM - Shuttle Radar Topography Mission (LEMOINE et al., 1998a; LEMOINE et al., 1998b; HENSLEY et al., 2001; JPL, 2004; FARR et al., 2007). O SRTM original tinha a altitude vinculada ao EGM96. Foi realizada uma atualização do modelo vinculando sua altitude ao EIGEN-GL04C, apresentando dessa forma melhor consistência no resultado final do modelo geoidal para América do Sul (BLITZKOW et al.,2008a,b). O mesmo foi usado para a obtenção da anomalia de gravidade completa de Bouguer, do efeito topográfico direto, do efeito topográfico indireto 
primário, do efeito topográfico indireto secundário, e do efeito atmosférico direto. Para isso foi utilizado o pacote computacional canadense SHGEO - Stokes-Helmert Geoid Software (VANÍČEK e KLEUSBERG, 1987; VANÍČEK e SJÖBERG, 1991; VANÍČEK e MARTINEC, 1994; VANÍČEK, 1995; VANÍČEK et al.,1999; ELLMANN, 2005a; 2005b). A malha de 5' das anomalias médias ar livre foi completada na região oceânica com as anomalias derivadas do modelo de altimetria por satélite do Danish National Space Center denominado DNSC08 (ANDERSEN et al., 2009). A componente de curto comprimento de onda foi estimada através do uso da transformada rápida de Fourier (FFT - Fast Fourier Transform) usando a modificação do núcleo de Stokes proposta por Featherstone (2003). O modelo do geopotencial EGM2008 (PAVLIS et al., 2008; BLITZKOW e MATOS, 2009), até o grau e ordem 150, foi utilizado como referência para remover a componente de longo comprimento de onda da anomalia de Helmert e para repor no final a mesma componente na altura geoidal, no que é denominado de técnica "remover-repor". A razão para a escolha do referido grau e ordem do EGM se trata de um modo de considerar coeficientes puramente baseados em observações do satélite e, portanto, não afetados pela distribuição irregular dos dados gravimétricos terrestres. $O$ MAPGEO2010 foi avaliado utilizando 844 referências de nível (RNs) que tiveram suas coordenadas geodésicas determinadas através de observações GPS, agora denominadas GPS/RN. Essas informações foram fornecidas pela Fundação IBGE. As alturas geoidais das mesmas foram obtidas a partir da associação das altitudes geodésica e ortométrica-normal, estas oriundas do nivelamento geométrico. Com o objetivo de identificar as melhorias obtidas no MAPGEO2010 em comparação com o seu antecessor, MAPGEO2004 (antigo modelo oficial de alturas geoidais no Brasil; IBGE, 2004; BLITZKOW e LOBIANCO, 2004; BLITZKOW et al., 2004).

\section{INFORMAÇÕES UTILIZADAS}

\subsection{Gravimetria terrestre}

O cálculo das alturas geoidais no Brasil exige dados gravimétricos no país e também nos países vizinhos. Diferentes organizações, universidades e institutos de pesquisa na América do Sul contribuíram com dados. É importante mencionar:

1. Observatório Nacional, Petrobras, Agência Nacional de Petróleo (ANP) e diversas universidades;

2. NGA (National Geospatial-Intelligence Agency);

3. GETECH Group plc;

4. 4BGI;

5. Instituições civis e militares dos países da América do Sul;

6. IBGE.

As Américas do Sul e Central contabilizam juntas 925.878 pontos gravimétricos. $\mathrm{O}$ atual modelo geoidal utilizou mais dados gravimétricos no seu cálculo do que o EGM2008, principalmente na Argentina, no centro-oeste e no 
sudeste do Brasil, no sul do Chile, na parte central do Equador e na região do Chaco no Paraguai (BLITZKOW et al., 2010; BLITZKOW e MATOS, 2009).

\subsection{Modelos do geopotencial}

Como mencionado (§1), o modelo do geopotencial (MG) utilizado como referência para o cálculo das alturas geoidais foi o EGM2008. O mesmo foi obtido pela combinação de dados do satélite GRACE (Gravity Recovery and Climate Experiment), EIGEN-GL04C (FÖRSTE et al., 2008) de grau e ordem 360, e dados gravimétricos terrestres e oceânicos. Ele foi calculado e disponibilizado para a comunidade científica pela equipe de desenvolvimento do Earth Gravitational Model (EGM) que pertencente a NASA (National Aeronautics and Space Administration) / NGA (PAVLIS et al., 2008). O modelo é completo até grau e ordem 2159 e contém coeficientes harmônicos esféricos adicionais até o grau 2190 e ordem 2159. Blitzkow e Matos (2009) avaliaram o mesmo para a América do Sul. Esse MG representa uma melhoria substancial na representação do campo gravitacional da América do Sul e apresenta um melhor resultado em termos estatísticos em comparação com MGs anteriores (EGM96, EIGEN-GL04S1, EIGEN-GL04C, GGM02S, GGM02C).

O MG GO_CONS_GCF_2_DIR, de grau e ordem 240, foi obtido usando a solução Direct Approach (DIR) desenvolvida pelo European GOCE Gravity consortium (EGGc). O referido modelo é o resultado da combinação das informações: ITG-Grace2010s até grau e ordem 150, dados de gravimetria terrestre, altimetria por radar e do gradiente de gravidade obtido pelo satélite GOCE (Gravity field and steady-state Ocean Circulation Explorer) (BRUINSMA et al., 2010; GRUBER et al., 2010; VISSER e IJSSEL, 2002).

GOCO02S é um projeto da ESA (European Spacial Agency) que tem como objetivo calcular MGs com alta acurácia e resolução. Ele é uma solução combinada dos satélites GOCE, GRACE, CHAMP (Challenging Minisatellite Payload) e SLR (Satellite Laser Ranging). O mesmo contém coeficientes dos harmônicos esféricos até grau e ordem 250 (GOIGINGER et al., 2011).

EIGEN 51C é um modelo do geopotencial global de ordem e grau 359 desenvolvido pelo GFZ (Deutsches GeoForschungsZentrum). O mesmo utilizou informação do campo gravitacional de seis anos de observação dos satélites CHAMP e GRACE (Outubro 2002 a Setembro 2008) e dados de anomalia de gravidade global do DNSC08 (BRUINSMA et al., 2010; ANDERSEN et al., 2009). EIGEN 06C é o MG mais recente do GFZ em cooperação com o Groupe de Recherche de Géodésie Spaciale de Toulouse. O mesmo é uma combinação das medições dos satélites LAGEOS (LAser GEOdynamics Satellite), GRACE e GOCE; como também de medições de gravimetria terrestres e dos dados da altimetria por satélite. $\mathrm{O}$ mesmo tem uma resolução espacial de aproximadamente $14 \mathrm{~km}$ (grau e ordem 1420) para o equador. 
A Figura 1 apresenta o erro de comissão dos modelos do geopotencial global em estudo. O erro de comissão reflete as incertezas dos próprios coeficientes harmônicos esféricos, que são devido a erros na observação que se propagam na ondulação geoidal. Esse erro para o grau máximo dos MGs é: $10 \mathrm{~cm}, 14 \mathrm{~cm}, 16 \mathrm{~cm}$, $9 \mathrm{~cm}$ e $8 \mathrm{~cm}$ para EIGEN 6C, EIGEN 51C, GOCO02S, GO_CONS_GCF_2_DIR_R2 e EGM08, respectivamente. O erro de comissão para o grau 150 (adotado para o MAPGEO2010) do EGM08 é de $6 \mathrm{~cm}$.

Figura 1 - Erro de comissão dos modelos do geopotencial globais.

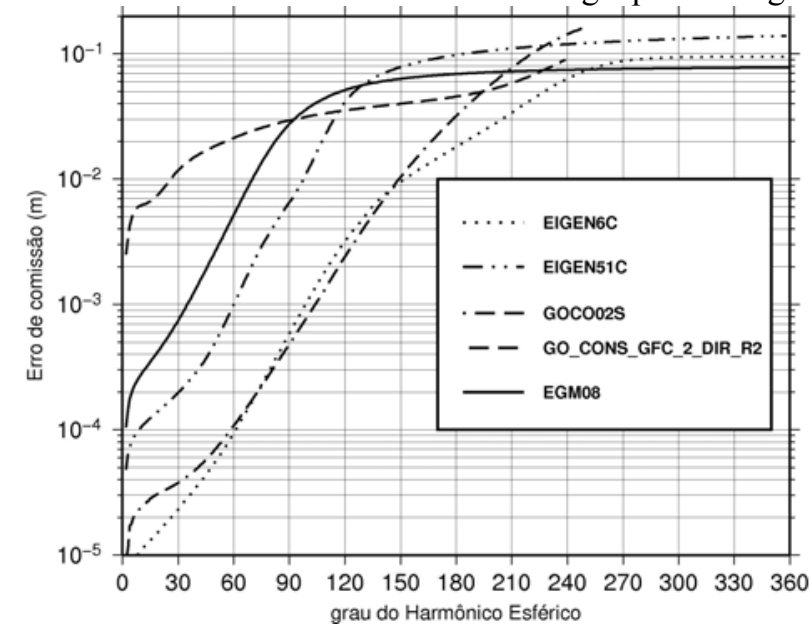

Foi considerado um termo de ordem zero (HOFMANN-WELLENHOF e MORITZ, 2006 pag. 111; HEISKANEN e MORITZ, 1967, p.103) igual a -0,41 m para o MAPGEO2010, EGM2008, GO_CONS_GCF_2_DIR_R2, GOCO02S, EIGEN 51C e EIGEN 6C. Este termo converte as alturas geoidais, que estão referenciadas a um elipsoide médio da Terra em valores que são referenciados ao WGS84; o mesmo foi estimado pela equipe do EGM2008. O referido elipsóide tem seus parâmetros no sistema livre maré, sendo: semi-eixo maior (a) igual a 6.378.136,58 $\mathrm{m}$ e achatamento (f) igual a 1/298,257686. O termo de ordem zero adotado para o modelo MAPGEO2004 foi de $-0,5 \mathrm{~m}$.

\subsection{Modelo Digital do Terreno}

A missão SRTM - Shuttle Radar Topography Mission foi um projeto conjunto da NASA - Estados Unidos, do DLR - Alemanha e da ASI - Itália utilizando a nave espacial Endeavour. Teve como um dos produtos o modelo digital do terreno (MDT) SRTM3 disponível a nível global (HENSLEY et al, 2001). Trata-se do MDT com uma resolução espacial de 3" de arco. A altitude, distância da nave ao solo, foi obtida através da técnica denominada interferometria por radar, onde uma 
antena emissora/receptora foi acoplada a nave espacial e uma segunda, somente receptora, fixada a uma distância de 60 metros da nave sobre uma haste retrátil. A altitude dos pontos foi determinada a partir da altitude geodésica_através do processamento dos dados do vôo e a altura geoidal obtida do EGM96 com grau e ordem 360 referenciado ao WGS84. O modelo possui diversos vazios em função de falhas ocorridas na obtenção dos dados (MATOS, 2005).

O LTG/EPUSP, desde os anos 90, desenvolve pesquisas no sentido de coletar e sistematizar dados altimétricos, os quais foram utilizados para preencher os vazios encontrados no SRTM3. Outra iniciativa conduzida no modelo SRTM3 foi a reconstituição das altitudes ortométricas substituindo o EGM96, originalmente utilizado, pelo modelo do geopotencial EIGEN-GL04C (FÖRSTE et al., 2008). A resolução espacial desse $\mathrm{MG}$ de grau e ordem 360 é de $0,5^{\circ}$. O resultado final foi a construção do modelo digital de terreno denominado SAM3s_v2, tendo uma área de abrangência em latitude de $25^{\circ} \mathrm{N}$ a $60^{\circ} \mathrm{S}$ e em longitude de $100^{\circ} \mathrm{W}$ a $25^{\circ} \mathrm{W}$ com resolução de 3 " de arco; o mesmo compreende um total de 1.983 quadrículas de $1^{\circ} \mathrm{x}$ $1^{\circ}$ (MATOS e BLITZKOW, 2008). Os pontos sem informação foram preenchidos por altitudes obtidas de cartas digitalizadas e do modelo DTM2002 (SALEH e PAVLIS, 2002; MATOS, 2005).

\subsection{Anomalias ar livre nas regiões oceânicas}

O Danish National Space Center lançou em 2008 o modelo de anomalia média ar livre denominado DNSC08GRA (ANDERSEN et al., 2009) em malha com resolução de 1'. O mesmo foi utilizado para completar a região oceânica da malha de 5' de anomalia média ar livre obtida através do pacote computacional SHGEO para a região continental. As anomalias foram obtidas a partir de altimetria por satélite utilizando dados altimétricos principalmente da missão ERS-1 e GEOSAT. Utilizaram-se dados também de outras missões como ICESAT, Topex/Poseidon, JASON-1, ERS-2, GFO ERM. Andersen et al. (2009) demonstram que esse modelo apresenta uma melhoria na precisão da ordem de $20-40 \%$ em relação aos modelos de gravidade globais marinhos anteriores, devido melhorias implantadas no processamento dos dados e a utilização do EGM2008 como superfície de referência para assegurar uma transição suave da gravidade do continente para o oceano (SANDWELL e SMITH, 2005 e 2009).

\section{DESENVOLVIMENTO TEÓRICO}

O esquema para a determinação do modelo geoidal pode ser resumido em 5 etapas (BLITZKOW et al., 2008):

1. Cálculo das anomalias pontuais ar livre através de dados terrestres de gravimetria (coordenadas, altitude ortométrica e aceleração de gravidade), (Figura 2);

2. Cálculo das anomalias completas de Bouguer, para posterior obtenção das anomalias médias ar livre em quadrículas de 5'. Para esses cálculos são necessárias as anomalias pontuais ar livre e um modelo digital de terreno; 
3. Cálculo das anomalias de gravidade de Helmert na superfície terrestre, que são obtidas adicionando à anomalia média ar livre, o efeito direto topográfico, o efeito atmosférico direto e efeito topográfico indireto secundário;

4. Integração de Stokes com a utilização da técnica "remover-repor";

5. Adição do efeito indireto topográfico primário nas alturas geoidais referidas a um "geoide fictício" denominado co-geoide (GEMAEL,1999; MATOS, 2005), para obtenção da alturas geoidais referidas ao novo geoide (Figura 3).

A determinação do geoide requer anomalias de gravidade sobre a Terra toda pela fórmula original de Stokes. Na prática, a área de integração é limitada pela vizinhança normalmente circular do ponto a ser calculado. A expressão de Stokes usada para a obtenção das alturas geoidais (ELLMANN e VANÍČEK, 2007) é:

$$
\begin{aligned}
\mathrm{N}(\Omega)= & \frac{\mathrm{R}}{4 \pi \gamma_{0}(\phi)} \iint_{\Omega_{\psi_{0}}} \mathrm{~S}^{\mathrm{M}}\left(\psi_{0}, \psi\left(\Omega, \Omega^{\prime}\right)\right) \Delta \mathrm{g}\left(\mathrm{r}_{\mathrm{g}}, \Omega\right) \mathrm{d} \Omega^{\prime}+ \\
& +\frac{\mathrm{R}}{2 \gamma_{0}(\phi)} \sum_{\mathrm{n}=2}^{\mathrm{M}} \frac{2}{\mathrm{n}-1} \Delta \mathrm{g}_{\mathrm{n}}^{\mathrm{h}}\left(\mathrm{r}_{\mathrm{g}}, \Omega\right)+ \\
& +\frac{\delta \mathrm{V}^{\mathrm{t}}\left(\mathrm{r}_{\mathrm{g}}, \Omega\right)}{\gamma_{0}(\phi)}+\frac{\delta \mathrm{V}^{\mathrm{a}}\left(\mathrm{r}_{\mathrm{g}}, \Omega\right)}{\gamma_{0}(\phi)}
\end{aligned}
$$

onde

$$
\Delta \mathrm{g}\left(\mathrm{r}_{\mathrm{g}}, \Omega\right)=\left(\Delta \mathrm{g}^{\mathrm{h}}\left(\mathrm{r}_{\mathrm{T}}, \Omega\right)-\sum_{\mathrm{n}=2}^{\mathrm{M}} \Delta \mathrm{g}_{\mathrm{n}}^{\mathrm{h}}\left(\mathrm{r}_{\mathrm{g}}, \Omega\right)\right)
$$

A posição geocêntrica $(r, \Omega)$ de qualquer ponto é representada pelo raio geocêntrico $\mathrm{r}$ e pelo par de coordenadas geocêntricas $\Omega=(\phi, \lambda) ; R$ é o raio médio terrestre. Neste trabalho, foi utilizado o núcleo de Stokes modificado $\mathrm{S}^{\mathrm{M}}\left(\psi_{0}, \psi\left(\Omega, \Omega^{\prime}\right)\right)$ proposto por Featherstone (2003), definido como uma combinação da modificação do núcleo de Stokes proposta por Vanİček e Kleusberg (1987) juntamente com a de Meissl (1971). Esse núcleo apresentou melhor resultado para a o cálculo do modelo geoidal, se comparado com a aplicação da técnica removerrepor com um kernel não modificado (LOBIANCO, 2005).

A modificação de Meissl (1971) propõe a simples subtração do valor da função núcleo de Stokes com relação à distância de truncamento, fazendo com que a série de Fourier do erro de truncamento convirja para zero mais rapidamente (FEATHERSTONE e SIDERIS, 1998). A modificação de Vaníček e Kleusberg (1987) trabalha para minimizar o erro de truncamento, isto é, usa a componente de baixa frequência da altura geoidal obtida pelo MG global e um esferóide de grau M como uma nova superfície de referência (VANÍČEK e SJÖBERG, 1991) ao invés do elipsoide de referência de Somigliana-Pizzeti (LOBIANCO, 2005). 
No lado direito da Equação 1, o primeiro termo é o co-geoide residual de Helmert. Como o campo de referência, com grau e ordem baixos, é removido antes da integração de Stokes (Equação 2), a contribuição do longo comprimento de onda precisa ser adicionada à componente residual da altura geoidal (o segundo termo do lado direito da Equação 1). A soma dos dois primeiros termos resulta no co-geoide de Helmert. O terceiro termo é o efeito topográfico indireto primário (MARTINEC, 1993) e o último termo é o efeito atmosférico indireto primário (NOVÁK, 2000). Ao se levar em conta os efeitos indiretos nas alturas geoidais obtem-se o quasigeoide.

O termo $\Delta \mathrm{g}^{\mathrm{h}}\left(\mathrm{r}_{\mathrm{t}}, \Omega\right)$, do lado esquerdo da Equação 2, é a anomalia de gravidade de Helmert referida à superfície da terra, que pode ser obtida por (VANÍČEK et al., 1999):

$$
\Delta \mathrm{g}^{\mathrm{h}}\left(\mathrm{r}_{\mathrm{t}}, \Omega\right)=\Delta \mathrm{g}\left(\mathrm{r}_{\mathrm{t}}, \Omega\right)+\delta \mathrm{A}^{\mathrm{t}}\left(\mathrm{r}_{\mathrm{t}}, \Omega\right)+\frac{2}{\mathrm{r}_{\mathrm{t}}(\Omega)} \delta \mathrm{V}^{\mathrm{t}}\left(\mathrm{r}_{\mathrm{t}}, \Omega\right)+\delta \mathrm{A}^{\mathrm{a}}\left(\mathrm{r}_{\mathrm{t}}, \Omega\right)
$$

O primeiro termo do lado direito da Equação 3 é a anomalia ar livre, o segundo e o terceiro termos são os efeitos topográficos direto e indireto secundário na atração gravitacional. $\mathrm{O}$ último termo é a correção não topográfica para a anomalia de gravidade de Helmert, denominado efeito atmosférico direto.

$\mathrm{O}$ efeito topográfico direto na gravidade $\left(\delta \mathrm{A}^{\mathrm{t}}\left(\mathrm{r}_{\mathrm{t}}, \Omega\right)\right)$ é uma quantidade residual. Este é obtido subtraindo a atração gravitacional das massas topográficas condensadas da atração das massas topográficas (ELLMANN e VANÍČEK, 2007). Analogamente, o efeito atmosférico direto (DAE) da gravidade $\left(\delta \mathrm{A}^{\mathrm{a}}\left(\mathrm{r}_{t}, \Omega\right)\right)$ é a atração gravitacional da atmosfera toda menos a atração gravitacional da atmosfera condensada (ELLMANN e VANÍČEK, 2007). O efeito topográfico indireto secundário origina-se devido à aplicação da correção topográfica na anomalia de gravidade. O seu valor numérico é sempre negativo e pequeno, podendo crescer ligeiramente em regiões montanhosas (MARTINEC e VANÍČEK, 1994; MARTINEC, 1998).

Para a obtenção das alturas geoidais utilizou-se o SAM_3sv2 e a malha de 5' x 5' das anomalias médias de gravidade ar livre, calculadas a partir de dados terrestres de gravimetria disponíveis para a área. $\mathrm{Na}$ região oceânica foram introduzidas as anomalias derivadas pelo DNSC08 (ANDERSEN et al., 2009). foi 150

O limite superior (M) usado para o núcleo de Stokes modificado (Equação 1)

\section{VALIDAÇÃO DO MAPGEO2010}

O novo modelo geoidal foi analisado através da utilização de 844 referências de nível, cujas altitudes ortométricas foram obtidas através de nivelamento geométrico (definido em um sistema de maré média) e que possuem altitudes 
geodésicas determinadas com GPS, sendo 484 pertencentes ao IBGE (Figura 4) e 360 ao banco de dados do Laboratório de Topografia e Geodésia (advindas também de outras instituições). As coordenadas geodésicas dos mesmos estão referidas ao SIRGAS2000, sistema livre de maré (FORTES, 2003). Devido às correções entre os sistemas de maré serem muito pequenas o IBGE não aplica as mesmas nas RNs. O objetivo da análise foi verificar a consistência entre as grandezas. Os desvios padrão das altitudes ortométricas, que passaram por novo ajustamento, e geodésicas dos pontos do IBGE são apresentadas nas Figuras 4 e 5. A Figura 6 mostra os desvios padrão em relação à latitude. Os desvios padrão das altitudes das RNs foram calculados e cedidos pelo IBGE (2011). Através desta figura verifica-se que os pontos têm precisão decimétrica na maioria do país. Os pontos do LTG podem ser considerados com precisão decimétrica para altitude. O método de interpolação bilinear foi utilizado para a obtenção das alturas geoidais nos referidos pontos a partir dos modelos em malha MAPGEO2010 e MAPGEO2004. Os dois modelos estão no sistema livre de maré. Em relação aos MGs as anomalias de altura adicionadas de um termo de correção dependente da topografia foram obtidas através dos coeficientes das funções harmônicas esféricas (BARTHELMES, 2009). Os valores estatísticos podem ser visualizados na Tabela 1 e a Figura 8 apresenta os histogramas das diferenças em intervalo de $0,2 \mathrm{~m}$. A porcentagem das discrepâncias entre $-1 \mathrm{~m}$ a $+1 \mathrm{~m}$ é de $99,64 \%, 89,93 \%, 98,70 \%, 92,42 \%, 98,46 \%, 98,46 \%, 98,81 \%$ e 99,05\% para os modelos MAPGEO2010, MAPGEO2004, EGM2008 (grau e ordem totais), EGM2008 $(\mathrm{n}=\mathrm{m}=150)$, GO_CONS_GCF_2_DIR_R2, GOCO02S, EIGEN 51C e 6C, respectivamente. O MAPGEO2010, o $\overline{\mathrm{EGM}} \overline{\mathrm{G}} 200 \overline{8}$ e o EIGEN 6C apresentaram melhores resultados estatísticos. Sendo o primeiro ligeiramente superior aos outros dois. A Figura 7 apresenta o mapa das discrepâncias entre os pontos GPS/RN e o MAPGEO2010.

Para avaliar a discrepância relativa dos modelos geoidais e do geopotencial foi possível selecionar 229 pares de pontos com distâncias entre 20 a $50 \mathrm{~km}$ dentre os 844 pontos GPS/RN. Aqueles estão localizados na maioria nas regiões sul e sudeste (Figura 9). Os outros pares restantes estão localizados no nordeste e no Estado de Tocantins (Figura 10). As figuras apresentam também a acurácia relativa em tons de cinza dos pares de pontos. Abaixo de $20 \mathrm{~km}$ as variações do modelo são pequenas e não permitem um bom juízo do mesmo e acima de $50 \mathrm{~km}$ a variação tende a não ser linear. Na Tabela 2 e na Figura 11 são apresentadas a análise estatística e histogramas da discrepância relativa dos 229 pares dos pontos GPS/RN, respectivamente. $\mathrm{O}$ importante nesse estudo é o resultado da acurácia relativa, que informa o desvio padrão dos pares de pontos estudados. O MAPGEO2010 apresenta o melhor resultado da análise. Pode-se observar que a contribuição dos curtos comprimentos de onda nesse modelo é extremamente relevante ao comparar o resultado da Tabela 2 e da Figura 11 do EGM2008 ( $\mathrm{n}=\mathrm{m}=150)$ usado como referência dos longos comprimentos de onda. 
Figura 2 - Distribuição de dados terrestres de gravimetria.

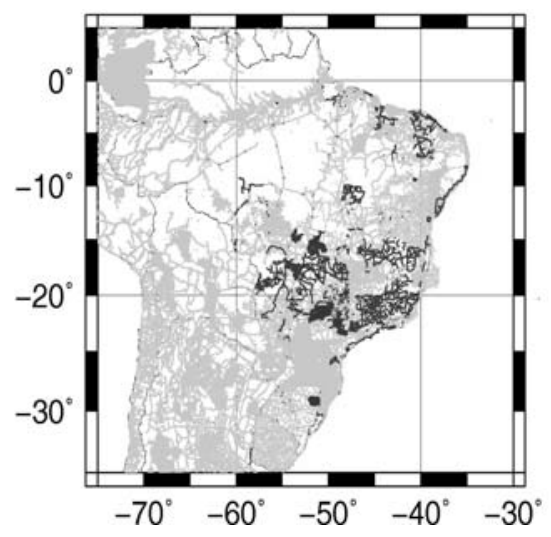

Figura 4 - Desvio padrão das altitudes ortométricas (484 pontos GPS/RN do IBGE).

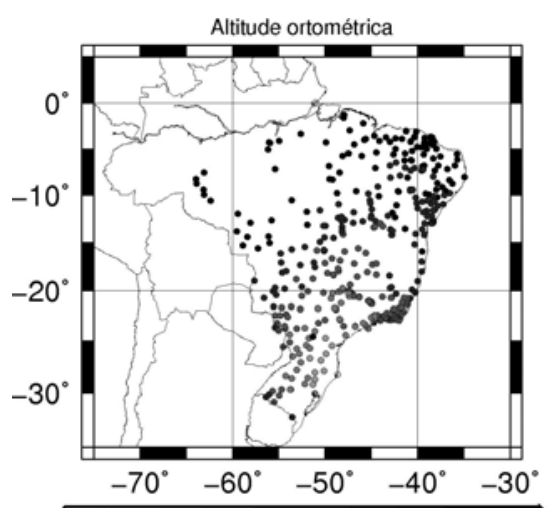

\begin{tabular}{llllllllllll}
\hline 0.00 & 0.01 & 0.02 & 0.03 & 0.04 & 0.05 & 0.06 & 0.07 & 0.08 & 0.09 & 0.10 \\
Desvio Padrăo (metro)
\end{tabular}
Figura 3 - Novo modelo de alturas geoidais para o Brasil.

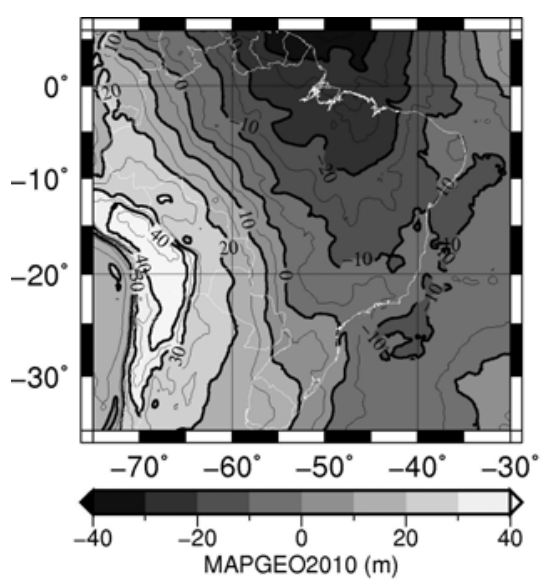

Figura 5 - Desvio padrão das altitudes geodésicas (484 pontos GPS/RN do IBGE).

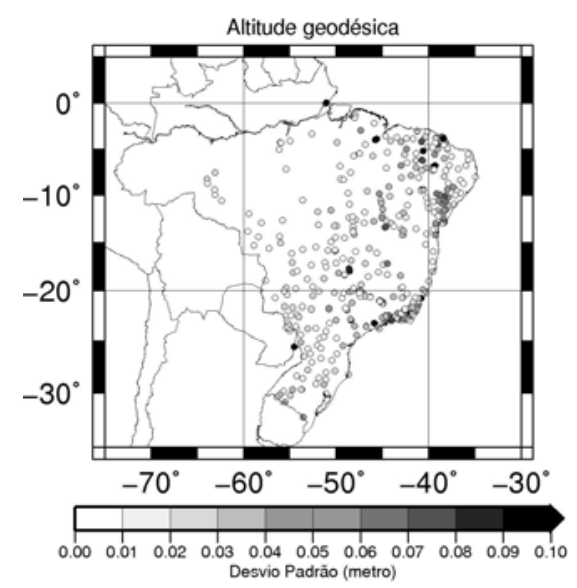


Figura 6 - Desvio padrão das altitudes ortométricas e geodésicas em função da Latitude (484 pontos GPS/RN do IBGE).

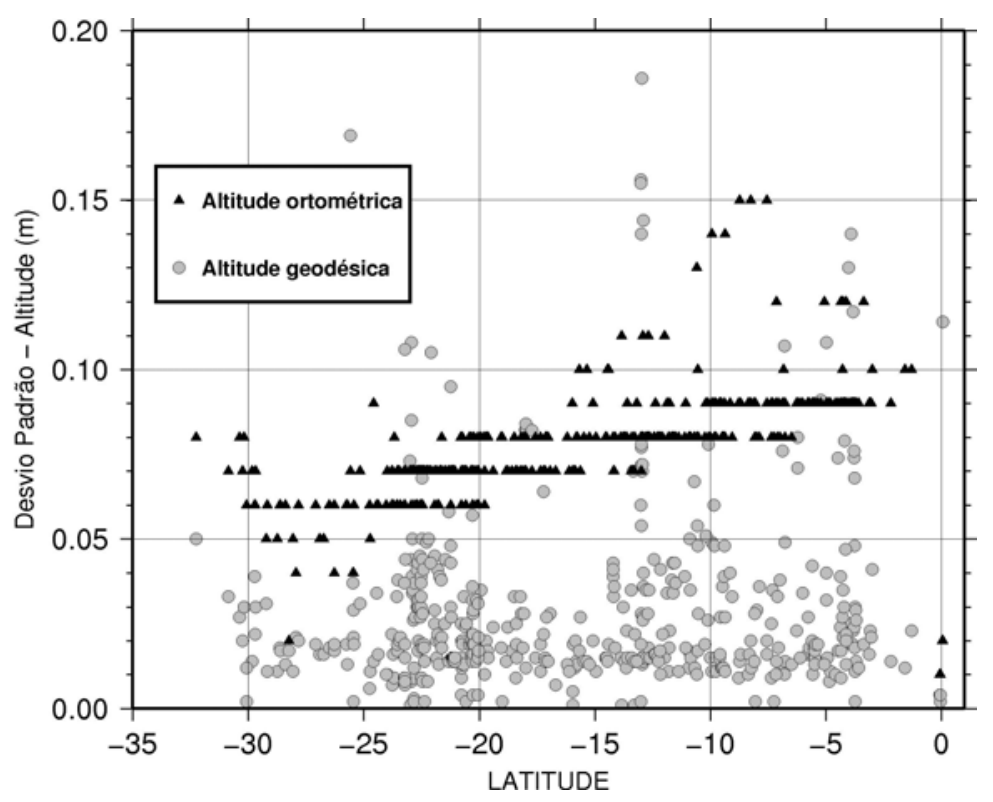

Figura 7 - Mapa das discrepâncias para o MAPGEO2010.

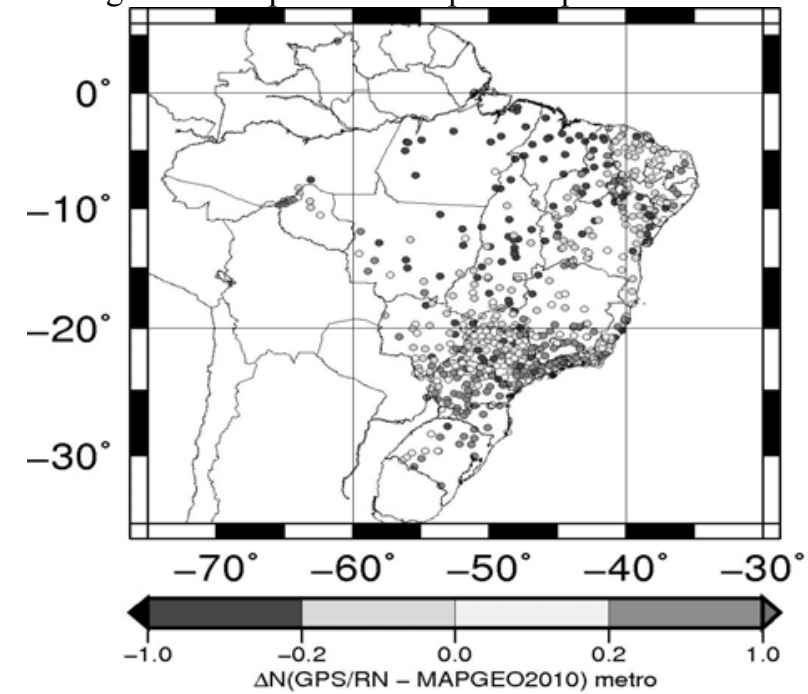

Bol. Ciênc. Geod., sec. Artigos, Curitiba, v. 18, nº 1, p.101-122, jan-mar, 2012. 
Figura 8 - Histogramas das discrepâncias absoluta em intervalos de $0,2 \mathrm{~m}$ para os
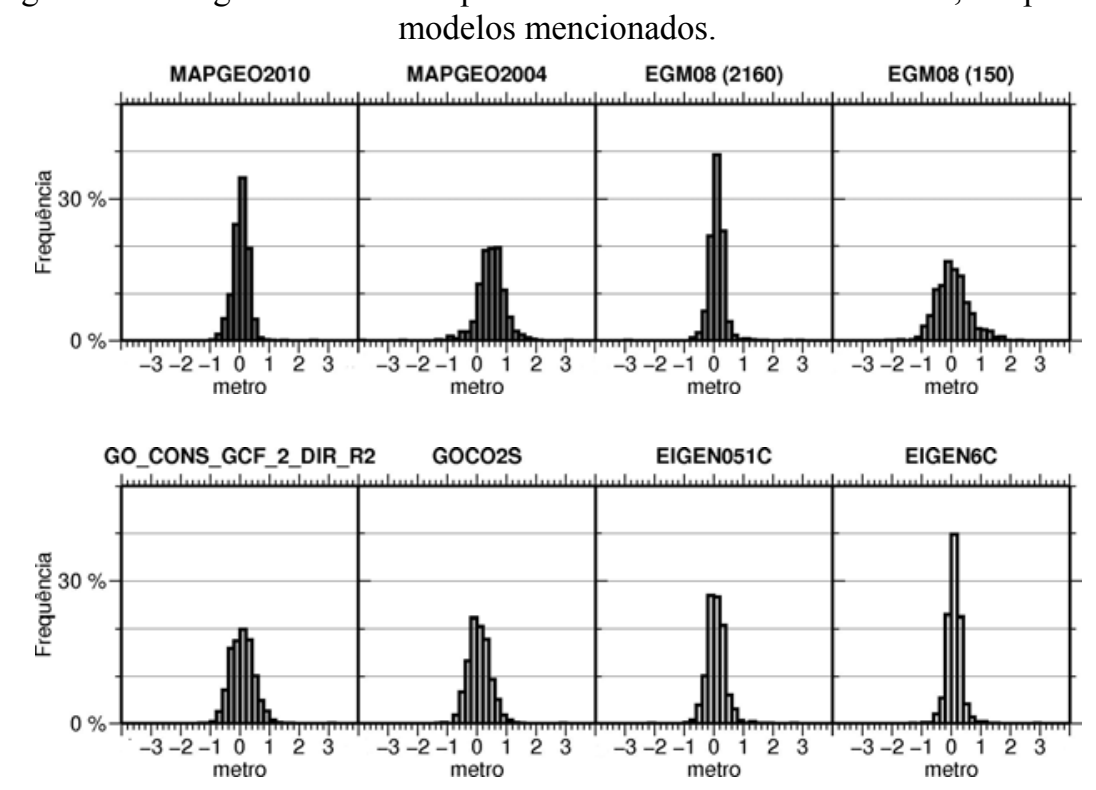

Tabela 1 - Análise estatística da discrepâncias absoluta utilizando GPS/RN no Brasil.

\begin{tabular}{l|c|c|c|c}
\hline & $\begin{array}{c}\text { Média } \\
\mathbf{( m )}\end{array}$ & $\begin{array}{c}\text { RMS } \\
\mathbf{( m )}\end{array}$ & $\begin{array}{c}\text { Máxima + } \\
\text { Discrepância } \\
\mathbf{( m )}\end{array}$ & $\begin{array}{c}\text { Máxima - } \\
\text { Discrepância } \\
\mathbf{( m )}\end{array}$ \\
\hline MAPGEO2010 & $\mathbf{0 , 0 4}$ & $\mathbf{0 , 2 8}$ & $\mathbf{2 , 4 7}$ & $\mathbf{- 0 , 9 3}$ \\
\hline MAPGEO2004 & 0,48 & 0,68 & 3,20 & $-3,84$ \\
\hline EGM2008 & $\mathbf{0 , 1 0}$ & $\mathbf{0 , 3 3}$ & $\mathbf{2 , 8 1}$ & $-\mathbf{2 , 9 3}$ \\
\hline EGM2008 (n=m=150) & 0,05 & 0,56 & 2,39 & $-2,10$ \\
\hline GO_CONS_GCF_2_DIR_R2 & 0.07 & 0,41 & 3,01 & $-1,29$ \\
\hline GOCO02S & 0,07 & 0,39 & 2,89 & $-1,30$ \\
\hline EIGEN 51C & 0,10 & 0,28 & 2,76 & -2.10 \\
\hline EIGEN 6C & 0,10 & 0,28 & 2,86 & $-1,39$ \\
\hline
\end{tabular}


Tabela 2 - Análise estatística da discrepância relativa dos pares de pontos GPS/RN.

\begin{tabular}{|c|c|c|c|c|}
\hline & $\begin{array}{c}\text { Média } \\
(\mathbf{c m} / \mathbf{k m})\end{array}$ & $\begin{array}{l}\text { Acurácia } \\
\text { Relativa } \\
(\mathbf{c m} / \mathbf{k m})\end{array}$ & $\begin{array}{c}\text { Máxima + } \\
\text { Discrepância } \\
(\mathbf{c m} / \mathbf{k m})\end{array}$ & $\begin{array}{l}\text { Máxima - } \\
\text { Discrepância } \\
\text { (cm/km) }\end{array}$ \\
\hline MAPGEO2010 & 0,12 & 0,77 & 2,85 & $-3,01$ \\
\hline MAPGEO2004 & 0,14 & 0,89 & 2,87 & $-2,58$ \\
\hline EGM2008 & 0,11 & 0,81 & 3,77 & $-3,81$ \\
\hline EGM2008 $(n=m=150)$ & $\mathbf{0 , 0 7}$ & 1,39 & 4,87 & $-5,67$ \\
\hline GO_CONS_GCF_2_DIR_R2 & 0,11 & 1,18 & 3,65 & $-4,26$ \\
\hline GOCO02S & 0,12 & 1,02 & 2,94 & $-3,95$ \\
\hline EIGEN 51C & 0,09 & 1,22 & 3,78 & $-4,01$ \\
\hline EIGEN 6C & 0,18 & 0,86 & 4,09 & $-1,73$ \\
\hline
\end{tabular}

Figura 9 - Pares de GPS/RN no Nordeste.

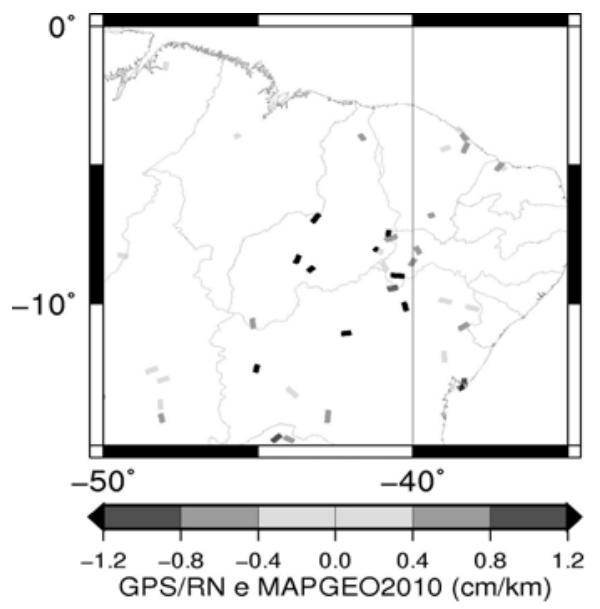

Figura 10 - Pares de GPS/RN no Sudeste.

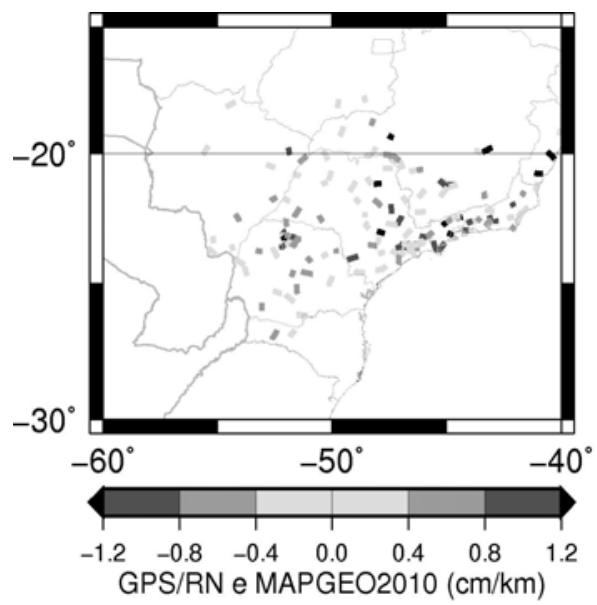


Figura 11 - Discrepância relativa dos pares de pontos GPS/RN
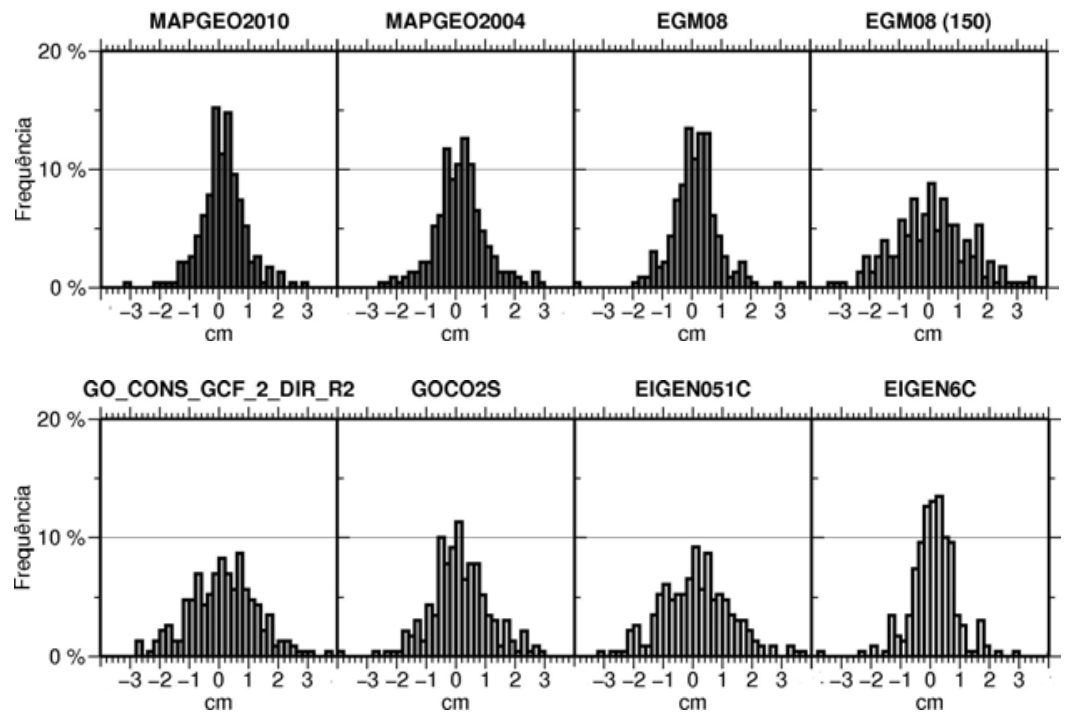

A região amazônica é coberta por rios e florestas o que torna impossível a realização do nivelamento geométrico para o estabelecimento de uma rede altimétrica. As linhas de nivelamento existentes medidas pelo IBGE não tem a precisão necessária no momento para a validação do modelo de alturas geoidais. Entretanto, trata-se de uma região acentuadamente plana e com gradiente pequeno nos rios. Como exemplo, a estação linimetrica de Tabatinga, que está situada no Rio Solimões e próxima à fronteira com o Peru, tem uma altura da superfície da água menor do que $70 \mathrm{~m}$ acima do nível médio do mar e está em torno de $3182,7 \mathrm{~km}$ da foz no Oceano Atlântico. Outro exemplo, a altura da superfície d'água durante a estação seca em Manaus é cerca de $12 \mathrm{~m}$ acima do nível médio do mar e está distante 1318,8 km da foz. Portanto, essas altitudes são uma fonte importante para a comparação de modelos geoidais (CAMPOS, 2004).

Campos (2004), em um trabalho conjunto com o IBGE, retratam o estabelecimento de 13 pontos de controle GNSS próximos às estações linimétricas localizadas no curso principal da bacia (rios Amazonas e Solimões). Essas estações são (Figura 12): Tabatinga (1); São Paulo de Olivença (2); Santo Antônio de Iça (3); Fonte Boa (4); C. Missões (5); Itapeuá (6);Manacapuru (7); P.Trapiche 15 (Manaus) (8); Parintins (9);Óbidos (10); Santarém (11); Porto de Moz (12); Porto de Santana (13).

A altitude ortométrica dos linimetros $\mathrm{H}_{\text {ZERO }}$ (zero da escala) é calculada pelas equações: 


$$
\begin{gathered}
\mathrm{H}_{\mathrm{GPS}-\mathrm{STA}} \sim \mathrm{h}-\mathrm{N} \\
\mathrm{H}_{\text {ZERO }}=\mathrm{H}_{\mathrm{GPS}-\mathrm{STA}} \pm \mathrm{dn}
\end{gathered}
$$

onde dn é a diferença de altura da linha de nivelamento medida entre o ponto GNSS (Estação SAT do IBGE) e o ponto de controle (estação linimetrica).

$\mathrm{O}$ valor do Nível da Água (NA) médio do mês adicionando o $\mathrm{H}_{\text {ZERO }}$ fornece a altitude da superfície da água (z).

$$
\mathrm{z}=\mathrm{H}_{\text {ZERO }}+\mathrm{NA}
$$

Esses pontos GNSS foram utilizados para verificar a consistência do MAPGEO2010 nos rios Amazonas e Solimões. Figura 13 apresenta a consistência do mesmo com relação à altura da superfície da água $(\mathrm{z})$ em função da distância do estuário dos rios principais para a média mensal de outubro/2008 (água baixa) e junho/2009 (água alta).

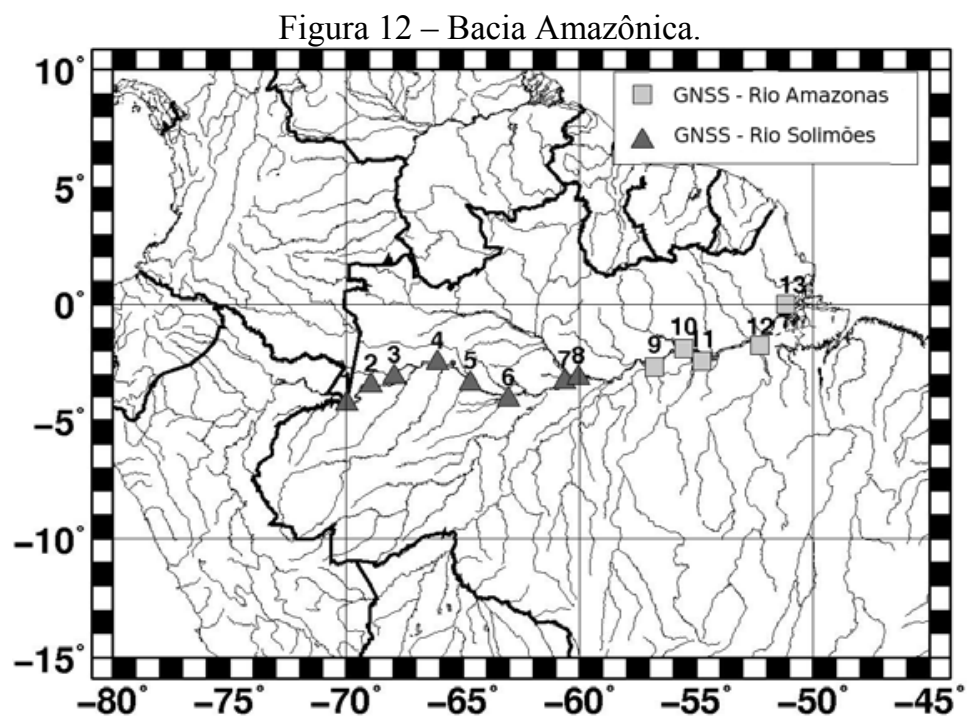

O gradiente médio desses rios é aproximadamente igual $20 \mathrm{~mm} / \mathrm{km}$ (COMPANHIA DE PESQUISA DE RECURSOS MINERAIS, 1999), desde Tabatinga até o oceano. Os gradientes médios obtidos pelo MAPGEO2010 para água alta e baixa foram de 20,7 e $18,4 \mathrm{~mm} / \mathrm{km}$, respectivamente. Portanto, o modelo ajusta-se muito bem à altura da superfície da água dos rios principais (Solimões e Amazonas). Com essa avaliação demonstra-se que o modelo MAPGEO2010 
também está bastante confiável para a região amazônica. O fato se deve à existência de dados gravimétricos ao longo dos rios levantados pela Petrobras na década de 50 .

Figura 13 - Altura da superfície da água (z) versus distância do estuário.

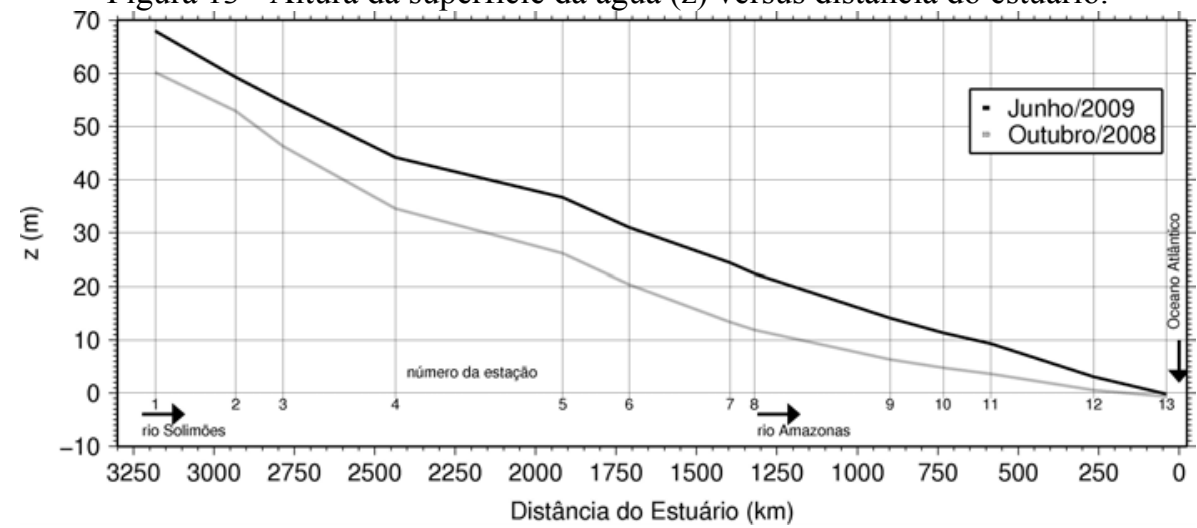

\section{CONCLUSÃO}

O novo modelo representa um avanço na qualidade das alturas geoidais no Brasil em relação ao MAPGEO2004. Isso ocorre devido ao trabalho intensivo nos últimos seis anos do IBGE em colaboração com a EPUSP-PTR e de várias instituições de pesquisa no Brasil e de outros países da América do Sul para suprir com informações gravimétricas regiões carentes. Além disso, as chamadas missões espaciais modernas, notadamente do satélite alemão de pesquisas geomagnéticas CHAMP (REIGBER et al., 2002a e 2002b) e dos satélites GRACE numa colaboração científica entre o GFZ - Deutsches GeoForschungsZentrum e a NASA (FOERSTE et al., 2008), permitiram uma melhoria nos modelos do geopotencial através da observação do campo gravitacional, o que significa uma adequação mais exata dos longos comprimentos de onda das alturas geoidais. Esta evolução fica comprovada na comparação dos dois modelos de alturas geoidais e dos cinco MGs com os pontos GPS/RN. É importante enfatizar que as alturas derivadas das observações GPS sobre RN não significam algo absoluto em precisão. Existe o erro do GPS e do nivelamento, sendo este mais crítico do que o primeiro (Figuras 4 a 6). Mas não deixa de ser uma informação que comprova a adequação e consistência das grandezas.

O estado brasileiro que apresenta as maiores discrepâncias dos pontos GPS/RN com o novo modelo é o Maranhão (Figura 7). Isto pode não significar uma degradação no modelo de alturas geoidais, mas uma possível inconsistência no nivelamento geométrico. 


\section{AGRADECIMENTOS}

Os autores agradecem às organizações civis e militares de diferentes países, ao Prof. Dr. Artur Ellmann (Universidade de Tecnologia de Tallinn, Estónia), Prof. Dr. Peter Vanicek e Prof. Dr. Marcelo Carvalho dos Santos (Universidade de New Brunswick, Canadá).

A atividade realizada teve o apoio financeiro do Governo do Canadá, através do Projeto Mudança do Referencial Geodésico Nacional que foi coordenado pelo Instituto Brasileiro de Geografia e Estatística (IBGE) e pela Universidade de New Brunswick (UNB) e patrocinado pela Agência Canadense para o Desenvolvimento Internacional (CIDA) através de um acordo internacional com a Agência Brasileira de Cooperação (ABC). O estado de São Paulo foi beneficiado com uma acentuada melhoria na distribuição de dados gravimétricos através do Projeto Temático "GNSS: investigações e aplicações no posicionamento geodésico, em estudos relacionados com a atmosfera e na agricultura de precisão", patrocinado pela FAPESP (Processo: 2006/04008-2).

\section{REFERÊNCIAS BIBLIOGRÁFICAS}

ANDERSEN, O.B.; KNUDSEN, P.; PHILIPPA, A.M.B. (2009). The DNSC08GRA global marine gravity field from double retracked satellite altimetry. Journal of Geodedy, 84:191-199, DOI 10.1007/s00190-009-0355-9.

BARTHELMES, F. (2009). Definition of Functionals of the Geopotential and Their Calculation from Spherical Harmonic Models. Scientific Technical Report STR09/02 do GFZ German Research Centre for Geosciences, 32p. Disponível em: http://icgem.gfz-potsdam.de/ICGEM/theory/str-0902.pdf. Acessado em 2011.

BLITZKOW, D.; MATOS, A.C.O.C.; GUIMARÃES, G.N.; LOBIANCO, M.C.B. (2010). Recent progress of the geoid in South America. $42^{\circ}$ Reunión del Consejo Directivo del Instituto Panamericano de Geografía e Historia (IPGH),Lima, Perú, 11 y 12 de noviembre de 2010. Disponível em: http://www.sirgas.org/fileadmin/docs/Boletines/Bol15/42_Blitzkow_et_al_Ge oid_in_South_America.pdf. Acessado em 2011.

BLITZKOW $\overline{\text {, D.; }}$ MATOS, A.C.O.C. (2009). EGM2008 and PGM2007A evaluation for South America. Newton's Bulletin, v. 4, p. 79-89. Disponível em: http://www.iges.polimi.it/pagine/services/newton/NewtonEGM2008/Report_A 3_SAmerica.pdf. Acessado em 2011.

BLITZKOW, D.; MATOS, A.C.O.C.; CAMPOS, I.O.; ELLMANN, A.; VANICEK, P.; DOS SANTOS, M. C. (2008a). An attempt for an Amazon geoid model using Helmert gravity anomaly. In: Michael G. Sideris. (Org.). Observing our Changing Earth. 1 ed. : Springer-Verlag, 2008, v. 133, p. 187-194. Disponível em: http://www.springerlink.com/content/p2560858820303t6/. Acessado em 2011.

BLITZKOW, D.; PACINO, M.C.; MATOS, A.C.O.C. (2008b). Activities in south America: Gravity and Geoid Projects. Disponível em: http://www. 
sirgas.org/fileadmin/docs/Boletines/Bol13/34_Activities_in_South_America Gravity_and_Geoid_Pacino_Blitzkow.pdf. Modelo disponível em: http://www.ptr.poli.usp.br/ltg/proj/proj26.htm. Acessado em 2011.

BLITZKOW, D.; LOBIANCO, M.C.B. (2004). Modelo Geoidal 2004 - Cálculo, resultados e comparações. Disponível em: http://www.ptr.poli.usp.br/ptr/siteant/FTP01/geoide2004.pdf. Acessado em 2011.

BLITZKOW,D.; LOBIANCO, M.C.B.; MATOS, A.C.O.C.; COGÔ DE SÁ, N. (2004). MAPGEO2004 - Projeto de mudança do Referencial Geodésico. Disponível em: http://www.ibge.gov.br/seminario_referencial_geocentrico/ portugues/arquivos/Blitzkow_01dez2004_Geoide_II_Seminario.pdf. Acessado em 2011.

BRUINSMA S.L.; MARTY J.C.; BALMINO G.; BIANCALE R.; FOERSTE C.; ABRIKOSOV O.; NEUMAYER H(2010). GOCE Gravity Field Recovery by Means of the Direct Numerical Method, presented at the ESA Living Planet Symposium, 27th June - 2nd July 2010, Bergen, Norway; Veja também: http://earth.esa.int/GOCE. Acessado em 2011.

CAMPOS, I.O. (2004). Referencial altimétrico para a Baciado Rio Amazonas. Tese de Doutorado - Escola Politécnica, Universidade de São Paulo, São Paulo, $112 \mathrm{p}$.

COMPANHIA DE PESQUISA DE RECURSOS MINERAIS (1999). Diagnóstico do Potencial Ecoturístico do Município de Monte Alegre. CPRM, Disponível em: http://www.cprm.gov.br. Acessado em 2011.

ELLMANN, A. (2005a). SHGEO software packages-An UNB Application to Stokes-Helmert Approach for Precise Geoid Computation, reference manual I, 36 p. Disponivel em: http://gge.unb.ca/Research/GRL/GeodesyGroup/ SHGeo_package/Manual/SHGeo_manual_I_v2.pdf. Acessado em 2011.

ELLMANN, A. (2005b). SHGEO software packages-An UNB Application to Stokes-Helmert Approach for Precise Geoid Computation, reference manual II, 43 p. Disponivel em: http://gge.unb.ca/Research/GRL/GeodesyGroup/ SHGeo package.

ELLMANN, A.; VANÍČEK, P. (2007). UNB application of Stokes-Helmert's approach to geoid computation, Journal of Geodynamics,43, 200-213.

FARR, T. G.; ROSEN, P. A.; CARO, E.; CRIPPEN, R.; DUREN, R.; HENSLEY, S.; KOBRICK, M.; PALLER, M.; RODRIGUEZ, E.; ROTH, L.; SEAL, D.; SHAFFER, S.; SHIMADA, J.; UMLAND, J.; WERNER, M.; OSKIN, M.; BURBANK, D.; ALSDORF, D. (2007), The Shuttle Radar Topography Mission, Reviews of Geophysics, 45, RG2004, doi:10.1029/2005RG000183.

FEATHERSTONE, W.E.; SIDERIS, M.G. (1998). Modified kernels in spectral geoid determination: first results from Western Australia. In: R. Forsberg and M. Feissl and R. Dietrich (Ed.). Geodesy on the move: gravity, geoids, geodynamics, and Antarctica, Berlin: Springer-Verlag. International Association of Geodesy Symposia , 119:188-193. 
FEATHERSTONE, W. E. (2003). Software for computing five existing types of deterministically modified integration kernel for gravimetric geoid determination, Computer \& Geosciences, 29, 183-193. Disponivel em: http://www.iamg.org/index.php. Acessado em 2011.

FOERSTE, C.; FLECHTNER, F.; SCHMIDT, R.; STUBENVOLL, R.; ROTHACHER, M.; KUSCHE, J.; NEUMAYER, K.-H.; BIANCALE, R.; LEMOINE, J.-M.; BARTHELMES, F.; BRUINSMA，J.; KOENIG，R.; MEYER, U. (2008), EIGEN-GL05C - A new global combined high-resolution GRACE-based gravity field model of the GFZ-GRGS cooperation, General Assembly European Geosciences Union (Vienna, Austria 2008), Geophysical Research Abstracts, Vol. 10, Abstract No. EGU2008-A-06944. Sítio do GRACE: http://op.gfz-potsdam.de/grace/results/grav/g007_eigen-05c.html. Acessado em 2011.

FORTES, L.P.S. (2003). O sistema de Referencia SIRGAS2000. XXI_Congresso_Brasileiro_de_Cartografia, Belo Horizonte, MG. Disponivel em: ftp://geoftp.ibge.gov.br/documentos/geodesia/pmrg/Apresentacao_em_ eventos/2003/XXI_Congresso_Brasileiro_de_Cartografia/CBC_2003_SIRGA S_A.pdf. Acessado em 2011.

GEMAEL, C. (1999). Introdução a Geodésia Física. Curitiba: $2^{\mathrm{a}}$ ed. Editora UFPR, $302 \mathrm{pp}$.

GOIGINGER H., HOECK E., RIESER D., MAYER-GUERR T., MAIER A., KRAUSS S., PAIL R., FECHER T., GRUBER T., BROCKMANN J.M., KRASBUTTER I., SCHUH W.-D., JAEGGI A., PRANGE L., HAUSLEITNER W., BAUR O., KUSCHE J. (2011). The combined satelliteonly global gravity field model GOCO02S. Presented at the 2011 General Assembly of the European Geosciences Union, Vienna, Austria, April 4-8.

GRUBER, T. VISSER, P. ACKERMANN, C. FECHER, T. HEINZE, M. (2010). Validation of GOCE Gravity Field Models and Precise Science Orbits. ESA Living Planet Symposium, Bergen, 2010.

HEISKANEN, W. A. AND MORITZ, H. (1967). Physical Geodesy, W. H. Freeman, San Francisco, CA, 364pp.

HENSLEY, S., MUNJY, R.; ROSEN, P. (2001). Interferometric synthetic aperture radar. In: Maune, D. F. (Ed.). Digital elevation model techonoligies applications: the DEM users manual. Bethesda, Maryland: ASPRS (The Imaging \& Geospatial Information Society), cap. 6, pp. 142-206.

HOFMANN-WELLENHOF, B.; MORITZ, H. (2006). Physical Geodesy (2nd. ed.). Springer, 403pp.

INSTITUTO BRASILEIRO DE GEOGRAFIA E ESTATÍSTICA (2004). Modelo de ondulação geoidal - MAPGEO2004. Disponível em: http://www.ibge. gov.br/seminario_referencial_geocentrico/portugues/arquivos/Lobianco_30nov 2004_Geoide_II_Seminario.pdf. Acessado em 2011.

INSTITUTO BRASILEIRO DE GEOGRAFIA E ESTATÍSTICA (2011). Ajustamento Simultâneo da Rede Altimétrica de Alta Precisão do Sistema 
Geodésico Brasileiro - Relatório, Disponível em: http:// ftp://geoftp.ibge. gov.br/documentos/geodesia/RelatorioAjustamento.pdf. Acessado em 2011.

JPL (2004). SRTM - The Mission to Map the World. Jet Propulsion Laboratory, California Inst. of Techn.. Disponivel em: http://www2.jpl.nasa.gov/srtm. Acessado em 2011.

LEMOINE, F.G.; PAVLIS, N.K.; KENYON, S.C., RAPP, R.H;PAVLIS, E.C.; CHAO, B.F. (1998a). New high-resolution model developed for Earth' gravitational field, EOS, Transactions, AGU, 79, 9, March 3, No 113, 117 118.

LEMOINE, F.G.; KENYON, S.C.; FACTOR, J.K; TRIMMER, R.G.; PAVLIS, N.K.; CHINN, D.S.; COX, C.M.; KLOSKO, S.M.; LUTHCKE, S.B.; TORRENCE, M.H.; WANG, Y.M.; WILLIAMSON, R.G;. PAVLIS, E.C.; RAPP R.H.; OLSON, T.R. (1998b). The development of the joint NASA GSFC and the National Imagery and Mapping Agency (NIMA) geopotential model EGM96, NASA/TP-1998-206861. National Aeronautics and Space Administration, Maryland, USA.

LOBIANCO, M.C.B. (2005) Determinação das alturas do geoide no Brasil. Tese de doutorado - Escola Politecnica, Universidade de Sao Paulo, São Paulo, 160p. http://www.teses.usp.br/teses/disponiveis/3/3138/tde-21022006-162205. Acessado em 2011.

MARTINEC, Z., (1993). Effect of lateral density variations of topographical masses in view of improving geoid model accuracy over Canada. Final Report of the contract DSS No. 23244-2-4356. Geodetic Survey of Canada, Ottawa.

MARTINEC, Z.; VANÍČEK, P. (1994). Direct topographical effect of Helmert's condensation for a spherical approximation of the geoid. Manuscripta Geodaetica, No. 19, pp. 257-268.

MARTINEC, Z. (1998). Boundary-value problems for gravimetric determination of a precise geoid. Lecture notes in earth sciences, 73, Springer.

MATOS, A.C.O.C.; BLITZKOW , D. (2008). Modelagem Digital de Terrenos (MDT) de 3" para a América do Sul (projeto de Pos-Doc). Dados disponíveis em: http://www.ptr.poli.usp.br/ltg/proj/proj26.htm. Acessado em 2011.

MATOS, A.C.O.C., 2005. Implementação de modelos digitais de terreno para aplicações na área de Geodésia e Geofísica na América do Sul. PhD thesis Escola Politécnica, Universidade de São Paulo, São Paulo, 355 p. Disponivel em: http://www.teses.usp.br/teses/disponiveis/3/3138/tde-10102005-104155. Acessado em 2011.

MEISSL, P. (1971). Preparations for the Numerical Evaluation of Second-Order Molodensky-Type Formulas. Ohio State University Report, 163, Columbus, USA.

NOVÁK, P., (2000). Evaluation of gravity data for the Stokes-Helmert solution to the geodetic boundary-value problem. Technical Report No. 207, Department of Geodesy and Geomatics Engineering, University of New Brunswick, Fredericton.

Bol. Ciênc. Geod., sec. Artigos, Curitiba, v. 18, nº 1, p.101-122, jan-mar, 2012. 
PAVLIS, N.K; HOLMES, S.A.; KENYON, S.C.; FACTOR, J.K. (2008). An earth gravitational model to degree 2160. Geophys Res (Abstract 10, E,GU2008-A01891, 2008, Ref ID: 1607-7962/gra/EGU2008-A-01891, EGU General Assembly). Disponivel em: http://earth-info.nga.mil/GandG/wgs84. Acessado em 2011.

SANDWELL, D. T.; SMITH, W. H. F. (2005), Retracking ERS-1 altimeter waveforms for optimal gravity field recovery. Geophysical Journal International, 163: 79-89. doi: 10.1111/j.1365-246X.2005.02724.x.

SANDWELL, D. T.; SMITH, W. H. F. (2009), Global marine gravity from retracked Geosat and ERS-1 altimetry: Ridge segmentation versus spreading rate, J. Geophys. Res., 114, B01411, doi:10.1029/2008JB006008.

SALEH, J.; PAVLIS; N.K. (2002). The development and evaluation of the global digital terrain model DTM2002, 3rd Meeting of the International Gravity and Geoid Commission, Thessaloniki, Greece.

REIGBER, CH.; LUEHR, H.; SCHWINTZER, P. (2002a). CHAMP Mission Status. Advances in Space Research, Vol. 30, No. 2, pp. 129-134. Sítio do CHAMP: http://www-app2.gfz-potsdam.de/pb1/op/champ/index_CHAMP.html. Acessado em 2011.

REIGBER, CH.; BALMINO, G.; SCHWINTZER, P.; BIANCALE, R.; BODE, A.; LEMOINE, J.-M.; KOENIG, R.; LOYER, S.; NEUMAYER, H.; MARTY,J.C.; BARTHELMES, F.; PEROSANZ, F.; ZHU, S. Y. (2002b). A high quality global gravity field model from CHAMP GPS tracking data and Accelerometry (EIGEN-1S). Geophysical Research Letters, 29(14), 10.1029/2002GL015064.

VANÍČEK, P.; KLEUSBERG, A. (1987). The Canadian geoid-Stokesian approach. Manuscripta Geodaetica, 12(2), pp. 86-98.

VANÍČEK, P.; SJÖBERG L.E. (1991), Reformulation of Stokes's theory for higher than second-degree reference field and modification of integration kernels, $J$. Geophys. Res. 96(B4), pp. 6339-6529. Disponivel em: http://www.agu. org/pubs/crossref/1991/90JB02782.shtml. Acessado em 2011.

VANÍČEK, P.; SJÖBERG, L.E. (1991), Reformulation of Stokes's theory for higher than second-degree reference field and modification of integration kernels, J. Geophys. Res. 96(B4), pp. 6339-6529.

VANÍČEK, P.; MARTINEC, Z. (1994). Compilation of a precise regional geoid. Manuscripta Geodaetica 19, pp 119-128. Disponivel em: http://gge.unb. ca/Personnel/Vanicek/StokesHelmert.pdf. Acessado em 2011.

VANÍČEK , P. (1995). Compilation of a precise regional geoid. Report for Geodetic Survey Division - DSS Contract: \#23244-1-4405/01-SS, Ottawa, pp.45. Disponivel em: http://gge.unb.ca/Personnel/Vanicek/GeoidReport 950327.pdf.

VANÍČEK, P.; J. HUANG, J.; NOVÁK, P.; PAGIATAKIS, S.D.; VÉRONNEAU, M.; MARTINEC, Z.; FEATHERSTONE, W.E. (1999). Determination of the boundary values for the Stokes-Helmert problem, J. Geodesy 73, pp. 160-192. 
Disponivel em: http://cat.inist.fr/?aModele=afficheN\&cpsidt=1787191. Acessado em 2011.

VISSER, P.N.A.M.; IJSSEL J. VAN DEN (2003). Aiming at a 1-cm orbit for Low Earth Orbiters:reduced-dynamic and kinematic precise orbit determination, Space Science Reviews, 108,27-36.

(Recebido em outubro de 2011. Aceito em fevereiro de 2012). 